www.jmscr.igmpublication.org

Impact Factor 5.84

Index Copernicus Value: 71.58

ISSN (e)-2347-176x ISSN (p) 2455-0450

crossref DOI: _https://dx.doi.org/10.18535/jmscr/v6i1.158

Journal Of Medical Science And Clinical Research

IGM Publication

An Official Publication of IGM Publication

\title{
A Comparison Study of QT Dispersion in Early and Delayed Thrombolytic Therapy in Acute ST Elevation Myocardial Infarction
}

Authors

Muralidharan Azhakesan ${ }^{1}$, Thanalakshmi Balachandran ${ }^{2}$, Sindhu Neelakandan $^{3}$

${ }^{1}$ Assistant Professor, Department of Cardiology, Kanyakumari Government Medical College Hospital

${ }^{2}$ OP Medical Officer, Dept of General Medicine, Kanyakumari Government Medical College Hospital

${ }^{3}$ Post Graduate, Dept of General Medicine, Kanyakumari Government Medical College Hospital

\begin{abstract}
Introduction: In analysis of Global burden of disease there is a shift from communicable to non communicable disease. Ischemic heart disease causes more death and disability than any other illness in the developed world.. With urbanisation, in countries with emerging economies, the prevalence of risk factors for IHD and the prevalence of IHD itself are both increasing ${ }^{(17)}$. Obesity, Insulin resistance and Type 2 Diabetus mellitus are Powerful risk factors for IHD. Population subgroups that appear to be particularly affected are men in South Asian countries, especially India and the Middle East. IHD is likely to become the most common cause of death worldwide by 2020. In United States 13 million persons have IHD and more than 7 million have sustained a Myocardial infarction ${ }^{(17) .}$

QTc and QTd are important parameters to predict mortality in patients with Acute STEMI. Many studies have evaluated the role of QTd and risk of Ventricular Arrhythmias in Acute STEMI. In this study we try to to emphasize that early thrombolytic therapy reduces QTd in Acute STEMI.

Methods: This study is conducted among 100 patients diagnosed with STEMI admitted in ICCU within 12 hrs of onset of symptoms at Kanyakumari Government medical college hospital .Patients with typical chestpain (more than 30 minutes),ST elevation >1mm in 2 or more limb leads, ST elevation > 2 mm in 2 or more precordial leads ,No contraindications for thrombolysis were included in the study.

Patients on drugs which prolong QT like Quinidine, Procainamide, Amiodarone, Sotalol ,TCA, Antihistamines astemizole, Azole Antifungals ,Drugs which reduce the $Q T$ like Digitalis, Patients with congenital long QT syndrome, Patients with Electrolyte disturbances, Patients with Atrial fibrillation, Bundle branch block, Patients with Acute carditis were excluded from the study.

Patients with STEMI admitted within 12hrs of onset of symptoms were enrolled, A standard 12 lead ECG was taken with paper speed of $25 \mathrm{~mm} / \mathrm{sec}$ at admission. Patients were treated with thrombolytic agent (streptokinase). Patients were divided in to patients treated with early thrombolysis within 3 hrs of onset of chest pain and lysed after $3 \mathrm{hrs}$ of onset of pain.,ECGs are taken after thrombolysis (90mins from the beginning of thrombolysis), after 24hrs and before discharge. QT, QTc,QTd, QTdc were calculated in patients admitted with STEMI and the difference of QT parameters in patients treated with early thrombolysis and in patients thrombolysed late due to delayed presentation were analysed

Results: In our study mean age of study subjects is 60.7 in early lysis group and 59.65 in late lysis group. Among 100 patients studied 40 patients were Diabetics, 36 patients were Hypertensives and 36 patients were smokers. Mean QT dispersion is $111 \mathrm{~ms}$ in early lysis group and $120 \mathrm{~ms}$ in late lysis group which is not significant statistically. Post lysis QT after 30mins of thrombolysis is $70 \mathrm{~ms}$ in Early lysis group and 110ms
\end{abstract}


in late lysis group which is statistically significant with the P value of 0.007. Mean QTd on the $2^{\text {nd }}$ day of thrombolysis is 60ms in early lysis group and 110ms in late thrombolysis group which is statistically significant with the P value of 0.0001. Mean QTd at the time of discharge is 50ms in early thrombolysis group and 100ms in late thrombolysis group which is statistically significant with the P value of 0.0001 .

In our study when the TIMI score is high the QT Maximum and QTd also high with a significant P value. When the TIMI score is low The QT value is also low with a significant $P$ value.

Conclusion: Markers of autonomic regulation of heart like QTd provides valuable information about the future course of events in a patient following acute STEMI which can be utilized to plan the future course of management in patients especially predisposed to adverse and catastrophic outcomes.

when the TIMI score is high the QT Maximum and QTd also high which predicts increased mortality

QTd significantly reduces in early and successful thrombolysis than late and failed thrombolysis which in turn prevent the risk of arrhythmias

Keywords: $Q T, Q T c, Q T d, Q T d c$, Acute STEMI, TIMI score.

\section{Introduction}

Acute myocardial infarction is one of the most common diagnoses in hospitalised patients in Intensive care unit. More than half of the Acute myocardial infarction related deaths occur before the striken individual reaches the hospital. The inhospital mortality rate after admission for AMI has declined from $10 \%$ to about $6 \%$. Mortality is approximately fourfold higher in elderly patients (over age 75) as compared with younger patients $^{(1)}$. The 12 lead electrocardiogram is a pivotal diagnostic and triage tool because it is at the center of the decision pathway for management. It permits distinction of those patients presenting with ST-segment elevation from those presenting without ST-segment elevation. Serum cardiac biomarkers are obtained to distinguish Unstable angina from non STsegment elevation myocardial infarction ${ }^{(17)}$

QTc and QTd are important parameters to predict mortality in patients with Acute STEMI. Many studies have evaluated the role QTd and risk of Ventricular Arrhythmias in Acute STEMI. In this study we try to emphasize that early thrombolytic therapy reduces QTd in Acute STEMI.

\section{Aims and Objectives}

- To calculate the QT,QTc,QTd,QTdc in patients admitted with STEMI and to analyse the difference of QT parameters in patients treated with early thrombolysis and in patients thrombolysed late due to delayed presentation
- To evaluate whether there is reduction in QT interval and QTd after early and successful thrombolysis which in turn reduces the risk of arrhythmias

\section{Materials and Methods}

\section{Study population}

This study is conducted among 100 patients diagnosed with STEMI admitted in ICCU within $12 \mathrm{hrs}$ of onset of symptoms at Kanyakumari Government medical college hospital

\section{Inclusion criteria}

- Patients with typical chestpain (more than 30 minutes)

- ST elevation $>1 \mathrm{~mm}$ in 2 or more limb leads, ST elevation $>2 \mathrm{~mm}$ in 2 or more precordial leads

- No contraindications for thrombolysis

\section{Exclusion criteria}

- Patients on drugs which prolong QT like Quinidine, Procainamide, Amiodarone, Sotalol

- TCA, Antihistamines astemizole, Azole Antifungals

- Drugs which reduce the QT like Digitalis

- Patients with congenital long QT syndrome

- Patiens with Electrolyte disturbances

- Patients with Atrial fibrillation, Bundle branch block

- Patients with Acute carditis 


\section{Methodology}

- Patients with STEMI admitted within $12 \mathrm{hrs}$ of onset of symptoms were enrolled

- A standard 12 lead ECG was taken with paper speed of $25 \mathrm{~mm} / \mathrm{sec}$ at admission

- Patients were treated with thrombolytic agent (streptokinase)

- Patients were divided in to patients treated with early thrombolysis within $3 \mathrm{hrs}$ of onset of chest pain and lysed after 3 hrs of onset of pain

- ECGs to be taken after thrombolysis (90mins from the beginning of thrombolysis), after $24 \mathrm{hrs}$ and before discharge.

- Successful thrombolysis is defined as ST segment resolution $>50 \%$ of the initial elevation

- $\quad$ The QT interval should be measured in all 12 leads

- The interval from the first deflection of QRS complex to the point of $\mathrm{T}$ wave to the isoelectric TP baseline

- The maximum slope intercept method is used to define the end of the $\mathrm{T}$ wave Bazett's formula: $\mathrm{QT}_{\mathrm{C}}=\mathrm{QT} / \sqrt{ } \mathrm{RR}$
- Framingham formula: $\mathrm{QT}_{\mathrm{C}}=\mathrm{QT}+0.154(1-$ RR)

- Bazett's formula is the most commonly used due to its simplicity. It over-corrects at heart rates $>100 \mathrm{bpm}$ and under-corrects at heart rates $<60 \mathrm{bpm}$, but provides an adequate correction for heart rates ranging from $60-$ $100 \mathrm{bpm}$.

- At heart rates outside of the $60-100 \mathrm{bpm}$ range, the Framingham corrections are more accurate and used instead.

- Patients are followed up for a period of 1 month for mortality and morbidity

- Interested patients are subjected to angiographic evaluation.

\section{Data collection}

- A detailed medical history and clinical examination will be done.

- Each patient will be assessed with TIMI score and Killip classification

\section{Investigations:}

Serum Electrolytes, CK-MB, 12 lead ECG, Echocardiogram

\section{Results}

Table 1: Age and sex distribution of Cases

\begin{tabular}{|l|c|c|c|c|c|c|c|}
\hline & $<30 y r s$ & $31-40 y r s$ & $41-50 y r s$ & $51-60 y r s$ & $61-70 y r s$ & $>70 y r s$ & Total \\
\hline male & 1 & 3 & 10 & 26 & 17 & 8 & 65 \\
\hline female & 0 & 0 & 4 & 8 & 16 & 7 & 35 \\
\hline$\leq 3 \mathrm{hrs}$ & 0 & 1 & 3 & 15 & 17 & 4 & 40 \\
\hline$>3.1 \mathrm{hrs}$ & 1 & 2 & 11 & 19 & 16 & 11 & 60 \\
\hline
\end{tabular}

Out of 100 cases thrombolysed 65 were males and 35 were females. Out of 100 cases thrombolysed

40 patients come under $\leq 3 \mathrm{hrs}$ group and 60 patients come under $>3$ hrs group

Table 2: Mean age of cases in Early and delayed thrombolysis group

\begin{tabular}{|l|c|c|c|c|c|}
\hline Group Statistics & & & & & \\
\hline & TIMWIN_G & $\mathrm{N}$ & Mean & Std. Deviation & P value \\
\hline AGE & $<3$ & 40 & 60.70 & 8.95 & 0.636 \\
\hline & $>3.1$ & 60 & 59.65 & 11.93 & \\
\hline
\end{tabular}

There is no significant difference in Mean age of patients in early and delayed thrombolysis group. 
Table 3: Risk factors for myocardial infarction:

\begin{tabular}{|l|c|c|c|c|}
\hline Risk factors & & $<3$ & $>3.1$ & Total \\
\hline \multirow{3}{*}{ DM } & No & 24 & 36 & 60 \\
\cline { 2 - 5 } & Yes & 16 & 24 & 40 \\
\cline { 2 - 5 } SHT & No & 22 & 42 & 64 \\
\hline \multirow{3}{*}{ SMOKING } & Yes & 18 & 18 & 36 \\
\cline { 2 - 5 } & No & 28 & 36 & 64 \\
\hline
\end{tabular}

In our study among 100 patients studied 40

patients were Diabetics, 36 patients were

Hypertensives and 36 patients were smokers.

\section{Chart:1}

\section{Types of MI included in the study}

25

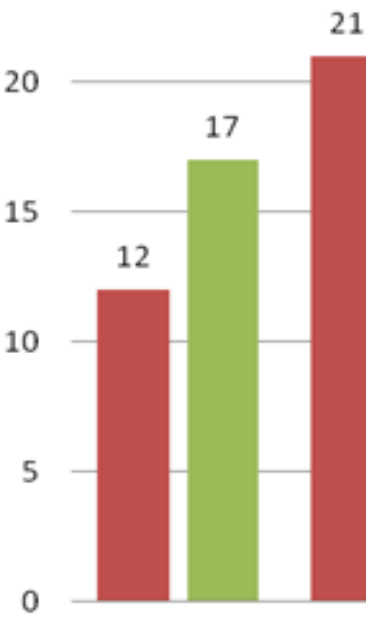

21
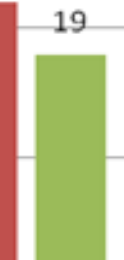

10

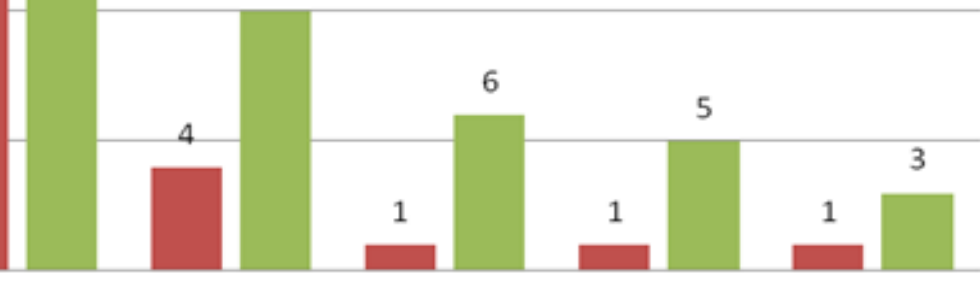

3

4

5

6

무 $<3.1$

In the present study group, the number of various myocardial infarction studied in both early and delayed thrombolysis group were almost similar.

Table 4: Mean QT Maximum and QTD dispersion in early thrombolysis group

\begin{tabular}{|l|c|c|c|c|}
\hline & & Mean & Std. Deviation & P value \\
\hline QT MAXIMUM & PRELYSIS & 0.50 & 0.04 & $<0.0001$ \\
\hline & POSLYSIS & 0.45 & 0.03 & \\
\hline QT MAXIMUM & PRELYSIS & 0.50 & 0.04 & $<0.0001$ \\
\hline & DAY2 & 0.43 & 0.06 & \\
\hline QT MAXIMUM & PRELYSIS & 0.50 & 0.04 & $<0.0001$ \\
\hline & AT DISCHARGE & 0.43 & 0.02 & \\
\hline QT DISPERSION & PRELYSIS & 0.11 & 0.02 & $<0.0001$ \\
\hline & POSLYSIS & 0.07 & 0.02 & \\
\hline QT DISPERSION & PRELYSIS & 0.11 & 0.02 & $<0.0001$ \\
\hline & DAY2 & 0.06 & 0.02 & \\
\hline QT DISPERSION & PRELYSIS & 0.11 & 0.02 & $<0.0001$ \\
\hline & AT DISCHARGE & 0.05 & 0.02 & \\
\hline
\end{tabular}

In early thrombolysis group, QT maximum and value of <.0001 on the day of thrombolysis, $2^{\text {nd }}$

QTd both were significantly reduced with the $\mathrm{P}$ day and on discharge. 


\section{JMSCR Vol||06||Issue||01||Page 32406-32416||January}

Chart 2: Mean QT maximum in Early thrombolysis group

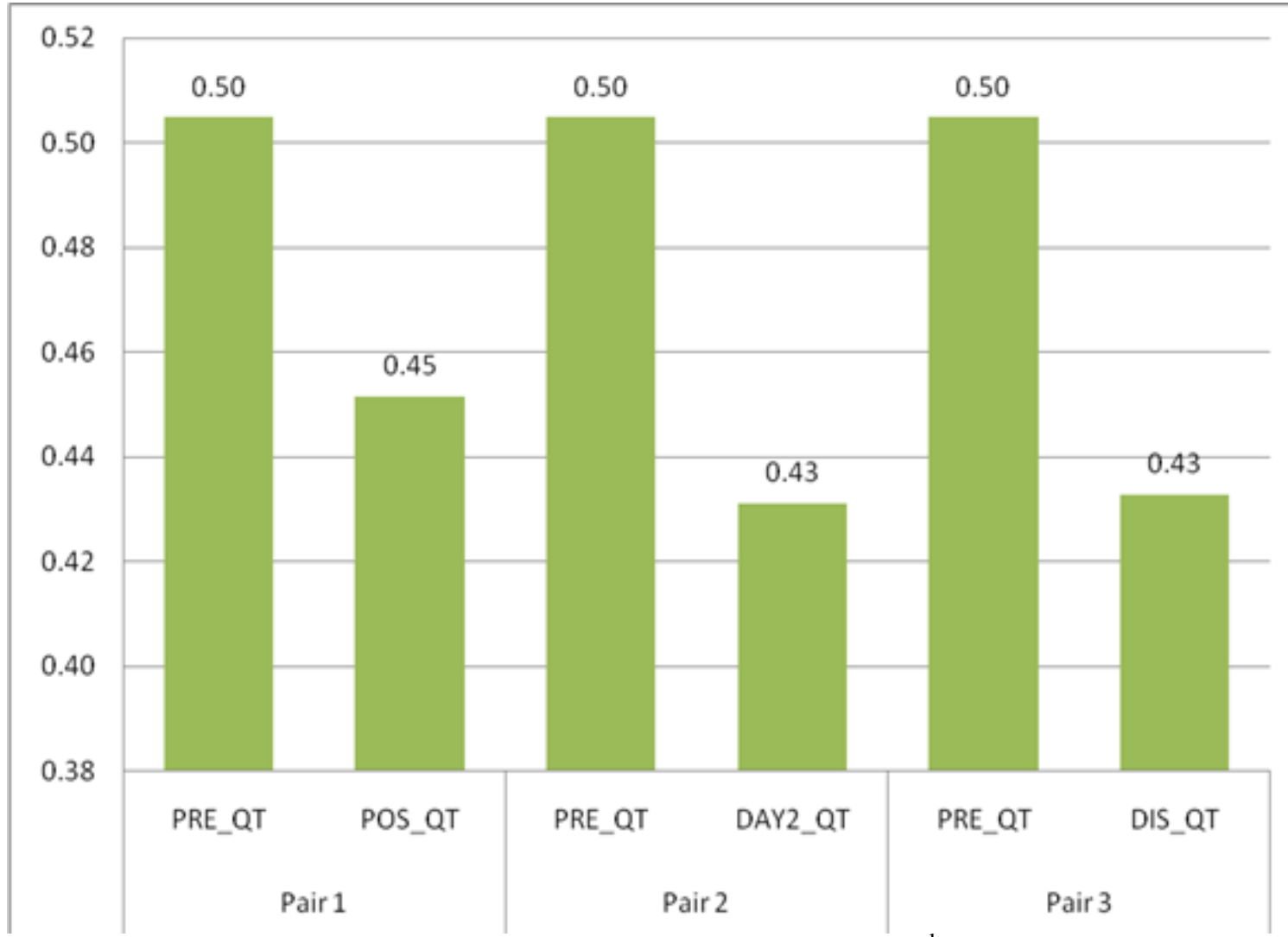

Mean QT Maximum is significantly lower after thrombolysis, on $2^{\text {nd }}$ day and at discharge in early thrombolysis group.

Chart 3: Mean QTd in Early thrombolysis group

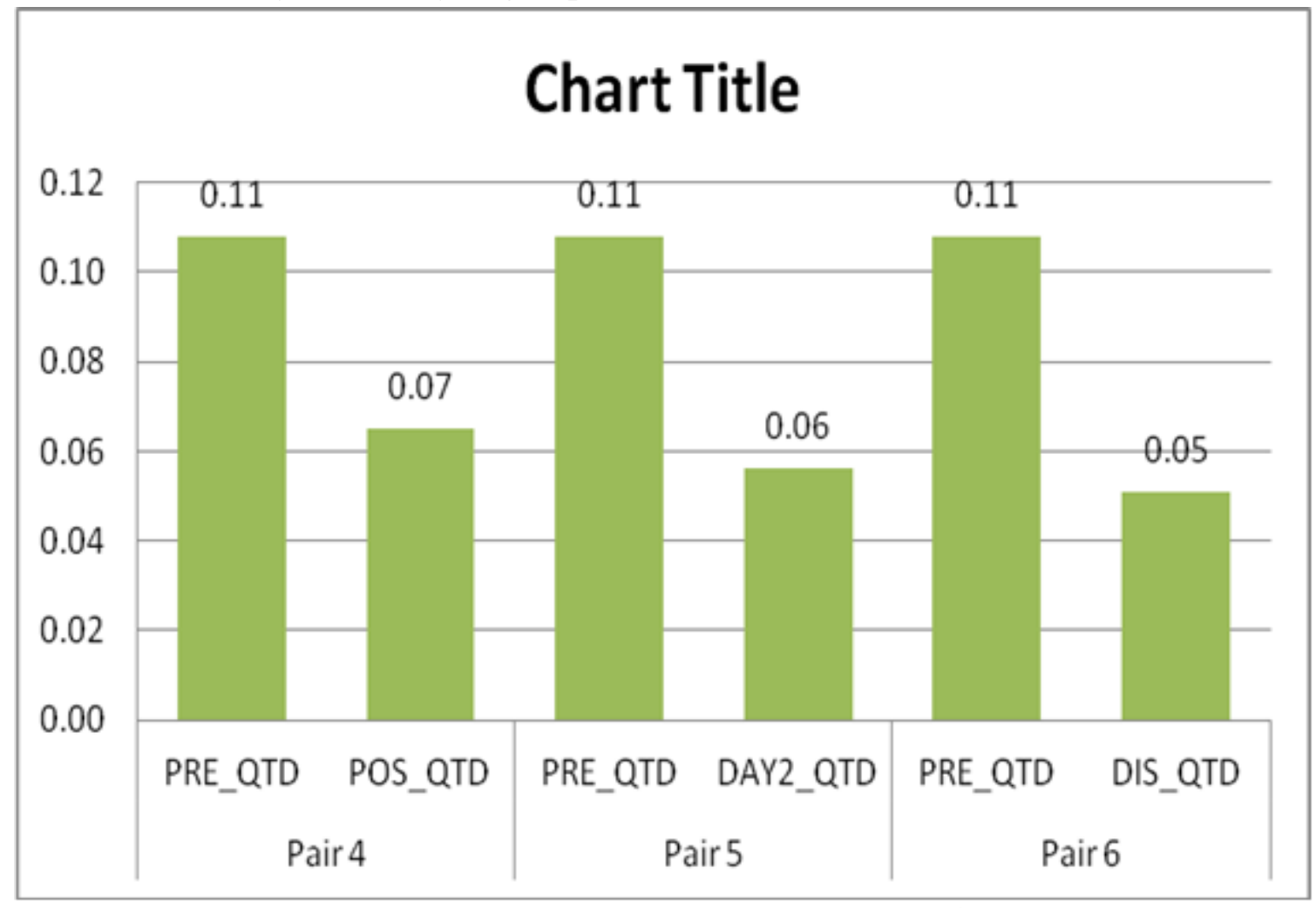

Mean QT dispersion is significantly lower after thrombolysis, on $2^{\text {nd }}$ day and at discharge in early thrombolysis group. 
Table: 5 Mean QT maximum and QTd in late thrombolysis group

\begin{tabular}{|l|c|c|c|c|}
\hline & & Mean & Std. Deviation & P value \\
\hline QT MAXIMUM & PRE LYSIS & 0.51 & 0.04 & 0.006 \\
\hline & POS LYSIS & 0.49 & 0.04 & \\
\hline QT MAXIMUM & PRELYSIS & 0.51 & 0.04 & $<0.0001$ \\
\hline & DAY2 & 0.49 & 0.03 & \\
\hline QT MAXIMUM & PRE LYSIS & 0.51 & 0.04 & 0.039 \\
\hline & AT DISCHARGE & 0.50 & 0.03 & \\
\hline & PRELYSIS & 0.12 & 0.05 & 0.866 \\
\hline QT DISPERSION & POS LYSIS & 0.11 & 0.11 & \\
\hline & PRE LYSIS & 0.12 & 0.05 & 0.254 \\
\hline QT DISPERSION & DAY2 & 0.11 & 0.06 & \\
\hline & PRELYSIS & 0.12 & 0.05 & 0.005 \\
\hline
\end{tabular}

QT maximum is significantly reduced in late thrombolysis group On the day of lysis, $2^{\text {nd }}$ day and at discharge. The Mean QTd is not significantly reduced on the day of thrombolysis and on the second day but significantly reduced at discharge

Chart 4: Mean QT Maximum in late thrombolysis group

\section{Chart Title}

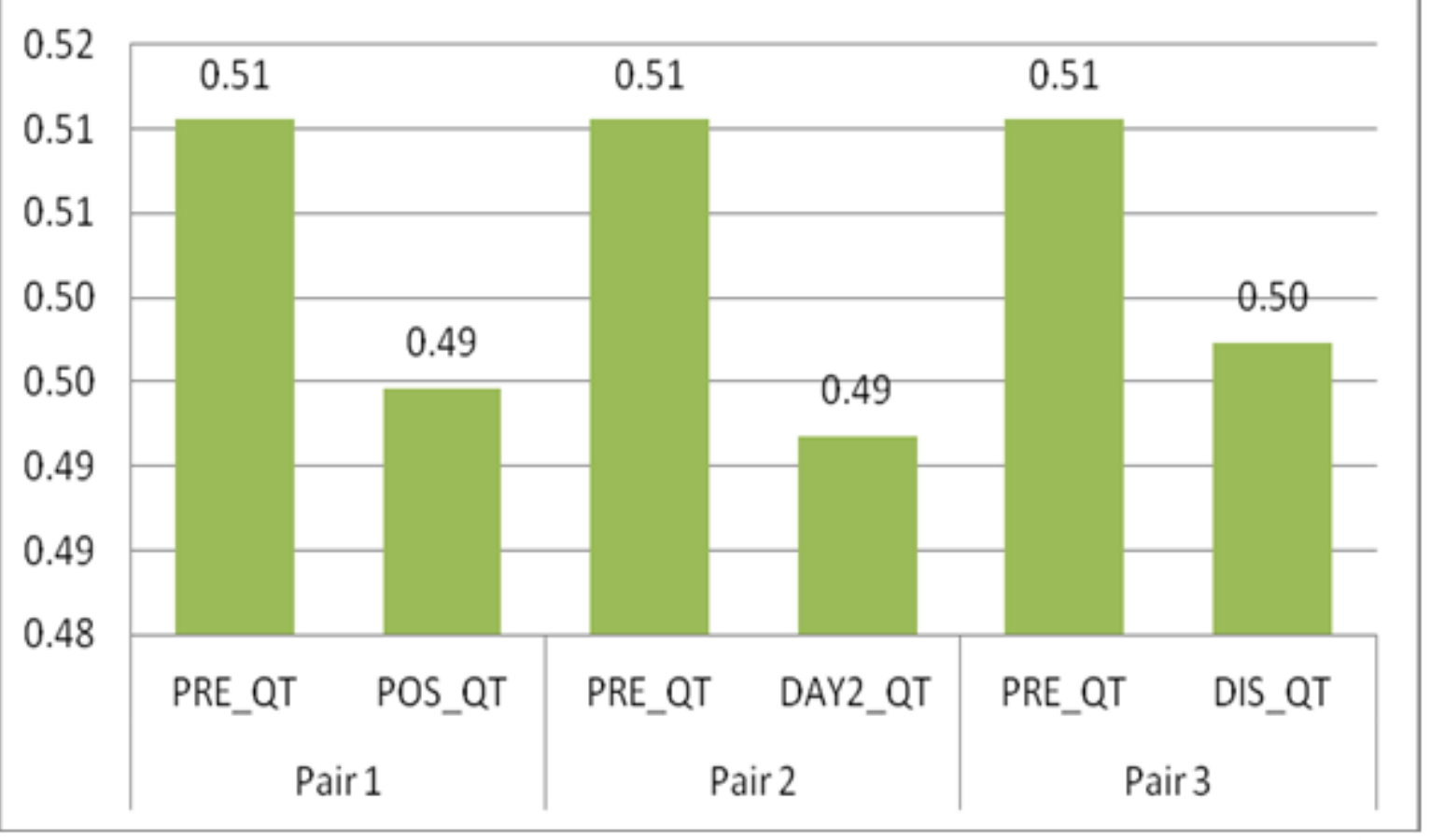

QT maximum is significantly reduced in late thrombolysis group On the day of lysis, $2^{\text {nd }}$ day and at discharge. 
Chart 5: Mean QTd in late thrombolysis group

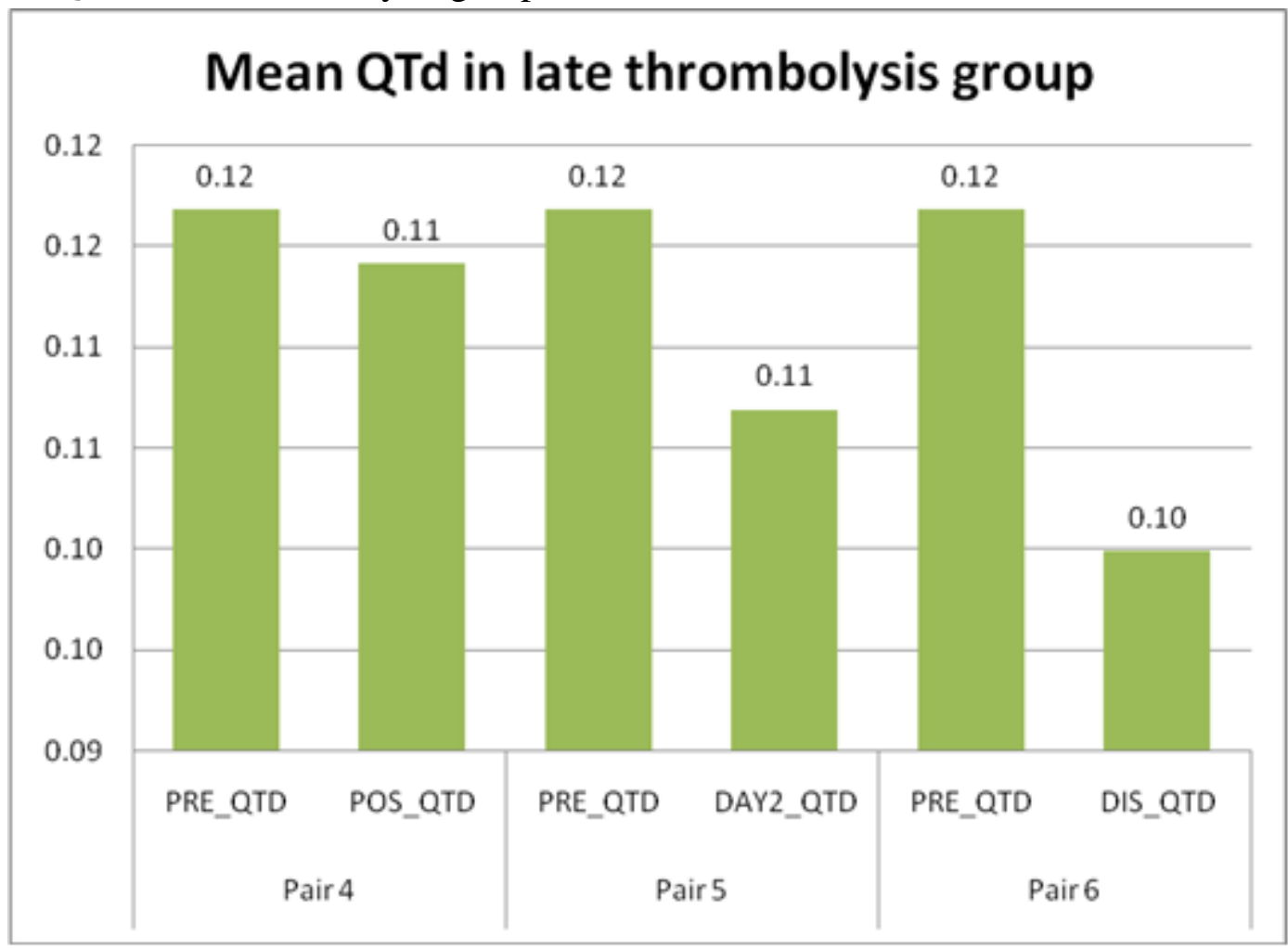

The Mean QTd is not reduced on the day of thrombolysis and on the second day but significantly reduced at discharge.

Table 6: Comparison of QT maximum and QTd in Early and Late thrombolysis group

\begin{tabular}{|l|c|c|c|c|}
\hline & TIMING & Mean & Std. Deviation & P value \\
\hline PRE_QT & $<3$ hours & 0.50 & 0.04 & 0.481 \\
\hline & $>3.1$ hours & 0.51 & 0.04 & \\
\hline PRE_QTD & $<3$ hours & 0.11 & 0.02 & 0.296 \\
\hline & $>3.1$ hours & 0.12 & 0.05 & \\
\hline POS_QT & $<3$ hours & 0.45 & 0.03 & $<0.0001$ \\
\hline & $>3.1$ hours & 0.49 & 0.04 & \\
\hline POS_QTD & $<3$ hours & 0.07 & 0.02 & 0.007 \\
\hline & $>3.1$ hours & 0.11 & 0.11 & \\
\hline DAY2_QT & $<3$ hours & 0.43 & 0.06 & $<0.0001$ \\
\hline & $>3.1$ hours & 0.49 & 0.03 & \\
\hline DAY2_QTD & $<3$ hours & 0.06 & 0.02 & $<0.0001$ \\
\hline & $>3.1$ hours & 0.11 & 0.06 & \\
\hline DIS_QT & $<3$ hours & 0.43 & 0.02 & $<0.0001$ \\
\hline & $>3.1$ hours & 0.50 & 0.03 & \\
\hline DIS_QTD & $<3$ hours & 0.05 & 0.02 & $<0.0001$ \\
\hline & $>3.1$ hours & 0.10 & 0.02 & \\
\hline
\end{tabular}

There is a significant reduction in QT Maximum in early lysis group than late lysis group on the day of thrombolysis, on the $2^{\text {nd }}$ day and at discharge.
There is a significant reduction in QTd in early lysis group than late lysis group on the day of thrombolysis, on the $2^{\text {nd }}$ day and at discharge. 


\section{JMSCR Vol||06||Issue||01||Page 32406-32416||January}

Chart 6: Comparison of QT maximum in Early and Late thrombolysis group:

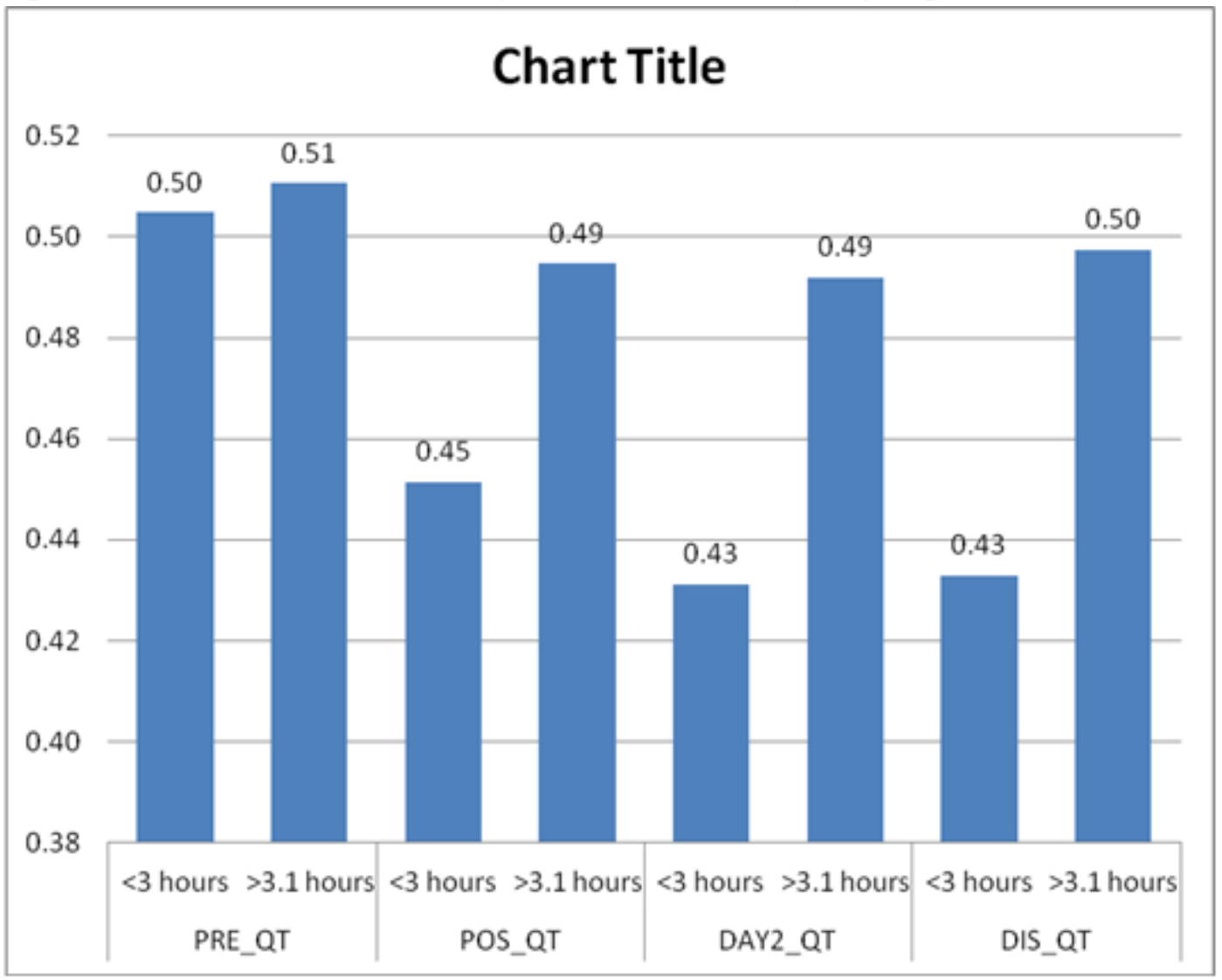

QT Maximum is significantly lower in Early and successful thrombolysis than late thrombolysis

Chart 7: Comparison of QTd in early and late thrombolysis group:

\section{Chart Title}

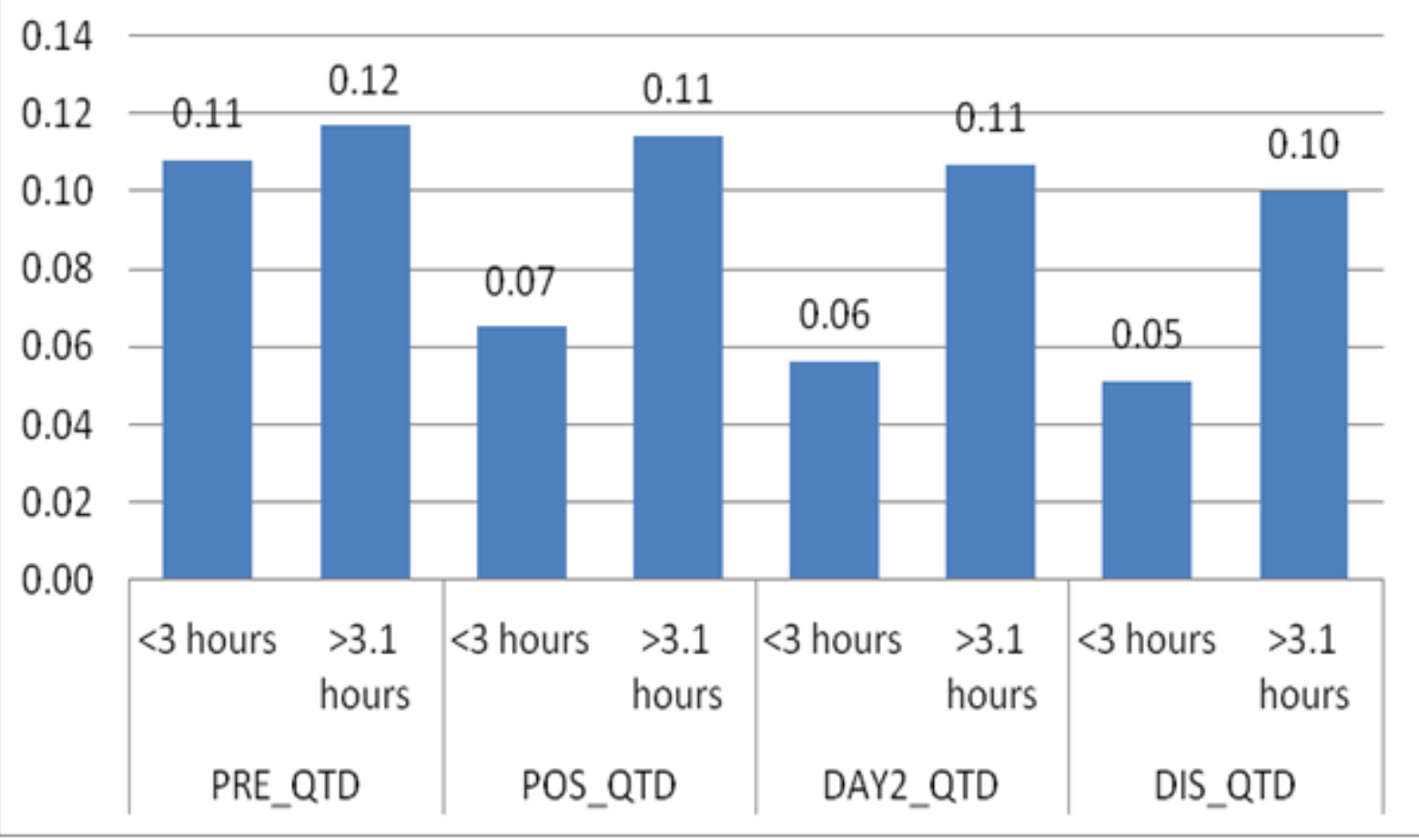

QTd is significantly lower in early and successful thrombolysis group than late thrombolysis group. 
Table 7: Correlation of QT Maximum, QTd with TIMI risk score

\begin{tabular}{|l|c|c|c|}
\hline & Correlation Coefficient & P value & $\mathrm{N}$ \\
\hline PRE_QT & 0.06 & 0.524 & 100 \\
\hline PRE_QTD & 0.08 & 0.440 & 100 \\
\hline POS_QT & 0.38 & $<0.0001$ & 100 \\
\hline POS_QTD & 0.28 & 0.005 & 100 \\
\hline DAY2_QT & 0.23 & 0.025 & 100 \\
\hline DAY2_QTD & 0.25 & 0.011 & 100 \\
\hline DIS_QT & 0.37 & $<0.0001$ & 100 \\
\hline DIS_QTD & 0.47 & $<0.0001$ & 100 \\
\hline
\end{tabular}

In all the cases studied, the QT maximum and QTd increases significantly as the TIMI score increases.

\section{Discussion}

Acute myocardial infarction represents one end of the spectrum of Coronary artery disease. About $50 \%$ of the deaths due to acute myocardial infarction occurs within 1 hour of the event and mainly attributable to arrhythmias. Qt dispersion has been suggested as one marker of automatic tone of the heart. QT dispersion reflects differences in the local myocardial repolarisation and hence the electrophysiological environment. Clinical interest in QTd on the surface ECG is based on the observation that regional heterogeneity of action potential in adjacent cardiac muscle tissue can initiate and sustain ventricular arrhythmias especially in vulnerable myocardium like that in Ischemic heart disease. Previous studies have proven that successful reperfusion decreases the QT dispersion and hence incidence of ventricular arrhythmias. So QT dispersion is a simple non invasive tool to assess the risk of arrhythmias after Acute myocardial infarction.

Our study compares the QTd in early and late thrombolysis group and the risk of arrhythmias.

In our study mean age of study subjects is 60.7 in early lysis group and 59.65 in late lysis group. Among 100 patients studied 40 patients were Diabetics, 36 patients were Hypertensives and 36 patients were smokers.

Mean QT dispersion is $111 \mathrm{~ms}$ in early lysis group and $120 \mathrm{~ms}$ in late lysis group which is not significant statistically.
Post lysis QT after 30mins of thrombolysis is $70 \mathrm{~ms}$ in Early lysis group and $110 \mathrm{~ms}$ in late lysis group which is statistically significant with the $\mathrm{P}$ value of 0.007

Mean QTd on the $2^{\text {nd }}$ day of thrombolysis is $60 \mathrm{~ms}$ in early lysis group and $110 \mathrm{~ms}$ in late thrombolysis group which is statistically significant with the $\mathrm{P}$ value of 0.0001 .

Mean QTd at the time of discharge is $50 \mathrm{~ms}$ in early thrombolysis group and $100 \mathrm{~ms}$ in late thrombolysis group which is statistically significant with the $\mathrm{P}$ value of 0.0001 .

Conclusions of study of Paventi S et al $1999^{28}$ was QT dispersion increased during acute MI.(2) The values were higher in the early hours, but decreased after thrombolytic therapy.(3) Greater QT dispersion is associated with severe ventricular arrhythmias

TEAM-2 Study Investigators have done a study on Reduction in QT interval dispersion by successful thrombolytic therapy in acute myocardial infarction ${ }^{29}$. They studied 244 patients with acute myocardial infarction (AMI) who were thrombolysed at an average of 2.6 hours after symptom onset.

There were significant differences in QTd $(96+/-$ $31,88+/-25,60+/-22$, and $52+/-19$ milliseconds; $\mathrm{P}<$ or $=.0001$,among TIMI perfusion grades $0,1,2$, and 3 , respectively.

In our study also the QTd is significantly lower after early and successful thrombolysis group with mean QTd prelysis was $110 \mathrm{~ms}, 70 \mathrm{~ms}, 60 \mathrm{~ms}, 50 \mathrm{~ms}$ in Prelysis, after $30 \mathrm{mins}$ of lysis and on $2^{\text {nd }}$ day and at discharge respectively. In late lysis group The mean QTd was $120 \mathrm{~ms}, 110 \mathrm{~ms}, 110 \mathrm{~ms}$ and 
$100 \mathrm{~ms}$ in Prelysis, after 30mins of thrombolysis, on $2^{\text {nd }}$ day and at discharge.

In our study we also observed that when the TIMI score is high the QT Maximum and QTd also high with a significant $P$ value. When the TIMI score is low The QT value is also low with a significant $\mathrm{P}$ value.

\section{Limitations of the Study}

1. Sample size was small so further studies with bigger sample size has to be done to further verify the results.

2. A limitation of QT interval assessment is that it is not always measurable in every lead or may be difficult to measure with precision in certain leads.

\section{Conclusion}

Markers of autonomic regulation of heart like QTd provides valuable information about the future course of events in a patient following acute STEMI which can be utilized to plan the future course of management in patients especially predisposed to adverse and catastrophic outcomes. when the TIMI score is high the QT Maximum and QTd also high which predicts increased mortality

QTd significantly reduces in early and successful thrombolysis than late and failed thrombolysis which in turn prevent the risk of arrhythmias

\section{Bibliography}

1. Chockalingam A, Balaguer-Vintro I: Impending Global Pandemic of cardiovascular diseases; challenges and opportunities for the prevention and control of cardiovascular disease in developing countries and economics in transition Borcelona, Prous Science 1999.

2. Haghjoo M, Kiani R, Fazelifar AF et al. Early risk stratification for arrhythmic death in patients with ST-Elevation Myocardial Infarction. Indian Pacing Electrophysiol J. 2007 Jan-Mar; 7(1): 1925.
3. Sahu P, Lim PO, Rana BS, Struthers AD.QT dispersion in medicine. Q J Med 2000; 93: 425-431.

4. Pye M, Quinn AC, Cobbe SM. QT interval dispersion: a non-invasive marker of susceptibility to arrhythmia in patients with sustained ventricular arrhythmias? $\mathrm{Br}$ Heart J 1994; 71: 511-514.

5. Parale GP, Adnaik AR, Kulkarni PM. Dynamics of QT dispersion in patients in Acute Myocardial Infarction. Indian Heart J 2003; 55(6): 628-31.

6. Day CP, McComb JM, Campbell RW. QT dispersion: an indication of arrhythmia risk in patients with long QT intervals. $\mathrm{Br}$ Heart J 1990; 63:342.

7. Schlant RC, Alexander RW, Ed "Diagnosis and management of chronic ischemic heart disease" Chapter No 58 in Hurst's - The Heart 8TH Edition Network.

8. Wilbur YW, Lew, Martin M LeWinter. Acute MI, Pathophysiology. Chapter-66: Kanu Chattergee et al. Cardiology an illustrated text reference. Vol.2: JB Lipincot Co. Philadelphia 7: 112pp.

9. Waller AD. A demonstration on man of electromotive changes accompanying the heart beat. J Physiol, 1887; 8.229.

10. Lewis T, Electrocardiology and its importance in the clinical examination of heart affections. III. The analysis of cardiac irregularities. Br Med J 1912;2: 65.

11. Parkinson J, Bedford De. Cardiac infarction and coronary thrombosis. Lancet 1928; $1: 4$.

12. Jervell A, Lange-Nielsen F. Congenital deaf-mutism, functional heart disease with prolongation of the QT interval, and sudden death. Am Heart J 1957; 54:59.

13. Romano and Ward. A new familial cardiac syndrome in children. Irish Med Association, 1964; 54,103.

14. Khaching Lehkan, Janos Malnar, John Sanberg "Relation of LV mass and QT dispersion in patients with systemic 
hypertension. "AM J Cardiol 1997; 79: 508 - 511.

15. M Pye et al, QT interval dispersion as a non invasive marker of susceptibility to ventricular arrhythmia. British heart journal. 1994; 71: 511-514.

16. Bazett HC. An analysis of the time relations of the electrocardiograms. Heart 1920; 7:353.

17. Harrison's principle of Internal Medicine 18th edition.

18. Cotran RS, Kumar V, Robbins SL (eds): Robbins Pathologic Basis of Disease. 5th ed. Philadelphia: WB Saunders, 1994.

19. Kloner RA, Jennings RB: Consequences of brief ischemia: Stunning, preconditioning, and their clinical implications: part Circulation 2001; 104:2981.

20. Matsuzaki M, Gallagher KP, Kemper WS, et al: Sustained regional dysfunction produced by prolonged coronary stenosis: Gradual recovery after reperfusion. Circulation 1983; 68:170.

21. Kloner RA, Jennings RB: Consequences of brief ischemia: Stunning, preconditioning, and their clinical implications: part Circulation 2001; 104:3158.

22. World Health Organization. World Health Statistics 2006:1-80. Available at: http://www.who.int/whosis/whostat2006.p df. Accessed August 15, 2006.

23. U.S. Department of Health and Human Services. The health consequences of smoking: a report of the surgeon general. Public Health Service Centers for Disease Control and Prevention, National Center for Chronic Disease Prevention and Health Promotion, Office on Smoking and Health. Atlanta, GA: USDHHS, 2004.

24. Thygesen K, Alpert JS, White HD. Joint ESC/ACCF/AHA/WHF Task Force for the Redefinition of Myocardial Infarction: Universal definition of myocardial infarction. Eur Heart J. 2007, 28: 2525-2538.
25. Rubin E, Farber JL (eds): Essential Pathology. 2nd ed. Philadelphia: JB Lippincott, 1995.

26. 26.Braunwald's heart disease : A textbook of cardiovascular medicine $10^{\text {th }}$ edition

27. Manual of cardiovascular medicine $4^{\text {th }}$ edition, Brian P Griffin

28. Paventi S, Bevilacqua U. Parafati MA, Di Luzio E, Rossi F, Pellieciom IIR. QT dispersion and early arrhythmic risk during acute myocardial infarction. Angiology. 1999;50:209-15..

29. FL Moreno, T Villanueva, LA Karagounis - Circulation, 1994 - Am Heart Assoc, Reduction in QT interval dispersion by successful thrombolytic therapy in acute myocardial infarction. TEAM-2 Study Investigators. 